\title{
A bioluminescence technique for quantitative and structure-associated imaging of pyruvate
}

\author{
Ulrike GA Sattler, Stefan Walenta and Wolfgang Mueller-Klieser \\ Institute of Physiology and Pathophysiology, University of Mainz, Mainz, Germany
}

\begin{abstract}
A novel bioluminescence assay has been developed for measuring pyruvate within sections of snap-frozen tissue in a quantitative manner as well as with a spatial resolution on a microscopical level. The assay was verified via HPLC and two independent photometric tests. The novel assay makes it possible to determine pyruvate concentrations in cryosections in the range of $0-5.0 \mu \mathrm{mol} / \mathrm{g}$ tissue (dry weight). Based on the analysis of samples of given pyruvate concentrations, the assay exhibits a recovery with a deviation $\leq 15 \%$. The minimal detectable amount was $0.02 \mathrm{pmol}$ based on a $20 \mu \mathrm{m}$ thick tissue section with an area of $1 \mathrm{~cm}^{2}$. Combination of the already established imaging bioluminescence techniques for ATP, glucose, and lactate with the novel pyruvate assay allows for a comprehensive characterization of the metabolic profile of individual tumors. As the redox state of cancer cells can be critical for the efficiency of irradiation and a number of chemotherapeutics, and as pyruvate and lactate are known to have radical scavenger functions, we hypothesize that the novel bioluminescence assay may be used for measuring the pretherapeutic lactate-to-pyruvate ratio which may predict the radiosensitivity of individual malignancies.

Laboratory Investigation (2007) 87, 84-92. doi:10.1038/labinvest.3700493; published online 27 November 2006
\end{abstract}

Keywords: bioluminescence; glycolysis; lactate; metabolite imaging; pyruvate; radical scavenger

Pyruvate is a core product of glycolysis which can be used to attain energy. Provided that sufficient oxygen is available, pyruvate is converted by pyruvate dehydrogenase (PDH) into acetylCoA which enters the citric acid cycle where it is metabolized to produce ATP in an oxidative way. Physiological concentrations of pyruvate range from 0.1 to $0.7 \mu \mathrm{mol} / \mathrm{g}$ in human skeletal muscle, from 30 to $260 \mu \mathrm{mol} / \mathrm{l}$ in blood, and from 80 to $170 \mu \mathrm{mol} / \mathrm{l}$ in cerebrospinal fluid. ${ }^{1-4}$ In some genetic diseases, such as PDH deficiencies, pyruvate concentrations can be increased up to $1 \mathrm{mmol} / \mathrm{l}$ in serum. ${ }^{5}$

The lactate-to-pyruvate ratio (L/P ratio) reflects the redox state of the cell and describes the balance between $\mathrm{NAD}^{+}$and $\mathrm{NADH}+\mathrm{H}^{+}$, which is dependent on the interconversion of lactate and pyruvate via lactate dehydrogenase $(\mathrm{LDH}) .{ }^{6}$ The normal $\mathrm{L} / \mathrm{P}$ ratio is $<20: 1$. An abnormal redox state can appear in a variety of situations, such as hypoxia, shock, sepsis, ischemia, and metabolic myopathies in which the $\mathrm{L} / \mathrm{P}$ ratio is often increased. According to the biochemical classification, metabolic myo-

Correspondence: Professor Dr W Mueller-Klieser, PhD, Institute of Physiology and Pathophysiology, University of Mainz, Duesbergweg 6, Mainz D-55099, Germany.

E-mail: wolfgang.mueller-klieser@uni-mainz.de

Received 4 July 2006; revised and accepted 21 September 2006; published online 27 November 2006 pathies are divided into glycogen storage diseases, lipid storage diseases, disorders of purine metabolism, and mitochondrial disorders. Eleven different subgroups of diseases caused by an underlying defect in glycogen synthesis, glycogenolysis, or glycolysis have been described. ${ }^{7}$ Most of these metabolic aberrations are primary innate defects of metabolism associated with aberrant enzymatic function due to defective genes, and all these disorders affect the musculoskeletal system. Anaerobic glycolysis is primarily involved during conditions of high-intensity isometric muscular activity. An enzyme deficiency in glycogen or glucose pathways could induce a bioenergetic crisis in muscle. For this reason, the $\mathrm{L} / \mathrm{P}$ ratio is an important diagnostic factor in metabolic myopathies to be registered. Quantitation of pyruvate in solutions (mostly acidic supernatants from biological samples) is possible via HPLC, ${ }^{8}$ photometric, ${ }^{9}$ or fluorimetric ${ }^{10}$ assays. All these techniques measure overall concentrations of pyruvate, but do not allow for the assessment of the metabolite distribution within complex biological tissues. At present, there are no commercial kits available for measuring pyruvate.

Our aim was to further exploit the versatility of the bioluminescence technique currently used for ATP, glucose, and lactate measurement ${ }^{11}$ by introducing quantitative pyruvate imaging. Hence, it will 
be possible to determine the relevant diagnostic $\mathrm{L} / \mathrm{P}$ ratio in tissues such as muscle biopsies from patients with metabolic myopathies without any chemical manipulation. Here, we show that the novel technique is suitable for the measurement of $\mathrm{L} / \mathrm{P}$ ratios in solid human tumors with the background of only sparse information on this ratio being available in literature. ${ }^{12}$ As the redox state of tumor cells might be critical for the efficiency of irradiation and a number of chemotherapeutics, and as lactate and pyruvate are known to have radical scavenger functions, ${ }^{10,13,14}$ it is likely that a correlation exists between the $\mathrm{L} / \mathrm{P}$ ratio and radiosensitivity of tumors. Data from others and our group ${ }^{15-17}$ lead us to the hypothesis that radiosensitivity of tumors may be predicted by measuring the pretherapeutic L/P ratio.

\section{Materials and methods}

\section{Assessment of Specimens}

When applying the bioluminescence technique in frozen tissue, adequate assessment of the specimens is one of the most crucial steps of the experimental procedure. Upon establishing the basic technique for ATP imaging, many methodological aspects of tissue freezing and handling of specimens have been investigated systematically and have been published. ${ }^{11}$ The most essential points will be briefly highlighted here. For solid tumors, it is sufficient to remove tissue by a rapid cut with a scalpel and to immerse the specimen into liquid nitrogen immediately. Minimization and standardization of the time between stoppage of blood perfusion and contact with nitrogen are most critical. This time should not exceed $30 \mathrm{~s}$ and should be invariant within one experimental series. Once the specimen is frozen, it has to be kept at liquid nitrogen temperature or in a $-80^{\circ} \mathrm{C}$ freezer until measurement. After sectioning in a cryomicrotome at $-20^{\circ} \mathrm{C}$, endogenous enzymes in the tissue section have to be heat-inactivated at $100^{\circ} \mathrm{C}$ for $10 \mathrm{~min}$. To illustrate the structureassociated metabolic imaging in this report, a frozen tissue specimen was chosen that contained parts of a solid tumor, adjacent skeletal muscle, and a layer of skin. The tumor was a human squamous cell carcinoma of the head and neck (HNSCC) which was grown in a nude mouse (courtesy of Michael Bauman, Technical University of Dresden, Germany).

\section{Biochemical Principle of Pyruvate-Dependent Bioluminescence}

The biochemical principle is based on the luminescence of luciferase from Photobacterium fischeri. The substrate of interest is linked to the NAD(P)/ $\mathrm{NAD}(\mathrm{P}) \mathrm{H}+\mathrm{H}^{+}$redox system through specific enzymes, and the redox system is then connected to the bacterial luciferase via FMN. In principle, it is possible to measure any substance that can be linked quantitatively to $\mathrm{NAD}(\mathrm{P}) / \mathrm{NAD}(\mathrm{P}) \mathrm{H}+\mathrm{H}^{+}$. This fact was utilized in the present study to design a novel procedure for measuring pyruvate within cryosections. The reactions involved in the detection of pyruvate by bioluminescence are depicted in Figure 1. Pyruvate is oxidatively decarboxylated to acetylCoA and $\mathrm{CO}_{2}$ via $\mathrm{PDH}$. Cofactors/coenzymes for the reaction are thiamine pyrophosphate (TPP), $\mathrm{CoA}$, and $\mathrm{NAD}^{+}$. The generated NADH can be linked to the light reaction in which initially $\mathrm{NADH}+\mathrm{H}^{+}$and FMN are converted to $\mathrm{FMNH}_{2}$ through the NADH:FMN-oxidoreductase (NFO). Luciferase catalyzes the bioluminescent oxidation of $\mathrm{FMNH}_{2}$ and a long-chain aliphatic aldehyde. In this process, emission of light occurs proportional to the initial pyruvate concentration.

\section{Pyruvate-Dependent Bioluminescence}

Enzyme solution

The enzyme solution for pyruvate-dependent bioluminescence measurements contained the following constituents: $50 \mathrm{mM}$ MOPS-Cl $\left(\mathrm{pH} 7.4\right.$ at $\left.30^{\circ} \mathrm{C}\right)$, $5 \mathrm{mM} \mathrm{MgCl}_{2}, 1 \mathrm{mM} \mathrm{CaCl}$, $2 \mathrm{mM}$ TPP, $0.25 \mathrm{mM} \mathrm{CoA}$, $6 \mathrm{mM} \mathrm{NAD}{ }^{+}, 3 \mathrm{mM}$ L-cysteine, $0.14 \mathrm{U} / \mathrm{ml} \mathrm{PDH,}$ $0.7 \mathrm{mM}$ FMN, $10 \mathrm{mM}$ decanal, $15 \mathrm{mU} / \mathrm{ml}$ luciferase, and $3 \mathrm{U} / \mathrm{ml}$ NFO. Chemicals were purchased from Sigma (Sigma-Aldrich, St Louis, MO, USA), enzymes from Roche (Roche Diagnostics $\mathrm{GmbH}$, Mannheim, Germany).

\section{Concentration standards}

Liver homogenates were used as standards for calibration of the bioluminescence reaction. A $150 \mathrm{~g}$ portion of porcine liver was cut into approximately $2 \mathrm{~cm}^{3}$ pieces and boiled for $40 \mathrm{~min}$ in $90^{\circ} \mathrm{C}$ heated destilled water. The pieces were mixed to a mushy consistency. This mass was homogenized completely in a tissue homogenizer (Braun, Melsungen, Germany). If necessary, PBS ( $\mathrm{pH} 7.4$ ) was used for liquefying the homogenate. Then, $3 \mathrm{~g}$ of liver

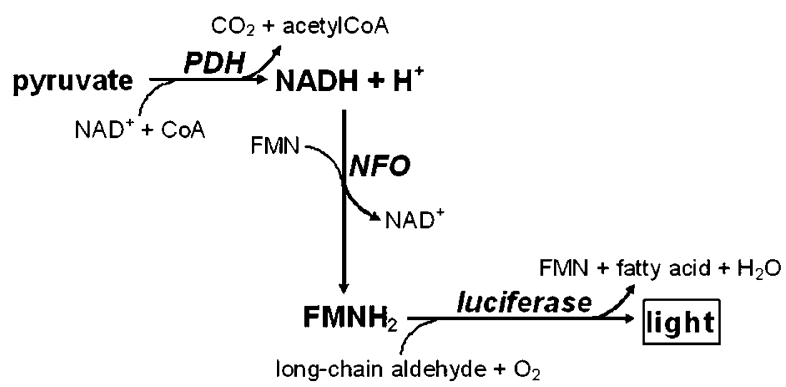

Figure 1 Biochemical reaction scheme of pyruvate-dependent bioluminescence. PDH uses pyruvate as substrate resulting in the production of $\mathrm{NADH}+\mathrm{H}^{+}$, which is linked to a light reaction via NFO and bacterial luciferase. Abbreviations: $\mathrm{PDH}$, pyruvate dehydrogenase; NFO, NADH:FMN-oxidoreductase; CoA, coenzyme A. 
homogenate were mixed with $0.5 \mathrm{ml}$ of pyruvate solutions with known content (standards) or a content blinded to the experimenter (reference samples). After vigorous vortexing, the pyruvate homogenates were sonified for $2 \mathrm{~min}$, put on ice for a few minutes and vortexed again. A measure of $1 \mathrm{~g}$ per standard or reference sample of homogenate was taken for HPLC and photometric measurements. Aliquots of the remaining homogenate were frozen and cut into $20 \mu \mathrm{m}$ cryosections for determination of pyruvate. Sections were adhered to coverglasses and fixed by heat for $10 \mathrm{~min}$ at $100^{\circ} \mathrm{C}$, which is identical to the processing of the actual tissue sections. Subsequently, sections were stored at $-20^{\circ} \mathrm{C}$ until bioluminescence measurement.

\section{Bioluminescence measurement procedure}

For bioluminescence imaging, coverglasses with adhered cryosections of standards, reference samples, or tissue were laid upside down on a metal slide with a casting mold. The mold was filled with the above-described enzyme solution for pyruvate or with that for lactate. ${ }^{11}$ The section was positioned in a way that it was in close contact with the solution. This 'sandwich' was transferred instantaneously to a thermostated reaction chamber on the stage of an appropriate microscope (Axiophot, Zeiss, Oberkochen, Germany). After $10 \mathrm{~s}$ incubation time at $30 \pm 1{ }^{\circ} \mathrm{C}$, bioluminescence was registered for a well-defined time interval. Emission of light was detected with a 16-bit CCD camera coupled to an imaging photon counting system (C2400, Hamamatsu, Herrsching, Germany) which was connected to the microscope. The whole assembly is located within a light-tight black box to prevent registration of background photons of the environment. For further details see Mueller-Klieser and Walenta, ${ }^{11}$ Walenta et al. ${ }^{16}$

\section{Image analysis}

The resulting bioluminescence images of the liver homogenate standards and reference samples were analyzed through Wasabi imaging software (Hamamatsu) and Origin data and graphic analysis software (OriginLab Corporation, Northampton, MA, USA). Images of the spatial distribution of light intensities in tissue sections were overlaid with images of sequential sections stained with hematoxylin and eosin (HE) to allow for separate data assessment in selected histological areas, such as viable tumor regions, stromal elements, necrosis, or tumor-adjacent normal tissue. For respective evaluation, the softwares Photoshop (Adobe Systems Incorporated, San Jose, CA, USA), ImageJ (National Institutes of Health, Bethesda, MD, USA), and Excel (Microsoft, Redmond, WA, USA) were used. Images were displayed in color codes for tissue concentration of the metabolites in units of $\mu \mathrm{mol} / \mathrm{g}$.

To generate geographical maps of $\mathrm{L} / \mathrm{P}$ ratios, images of lactate and pyruvate concentration values obtained in two sequential cryosections were overlaid precisely, and each pixel value for lactate was divided by its corresponding pyruvate pixel value. The resulting spatial distribution of $\mathrm{L} / \mathrm{P}$ ratios was then converted into a color-coded image.

For statistical evaluation of the images, frequency distribution histograms were generated from the pixel values for lactate, pyruvate, and $\mathrm{L} / \mathrm{P}$ ratios. This was performed for the whole tissue section and, separately, for tumor regions, muscle and skin which were all contained in the frozen specimen considered.

\section{Non-Bioluminescent Pyruvate Assays}

Determination of pyruvate via non-bioluminescent assays was performed with perchloric acid extracts. One gram of liver homogenate from standards and reference samples was deproteinized by addition of $0.2 \mathrm{ml}$ of $5 \%$ perchloric acid remaining on ice for $30 \mathrm{~min}$. After vigorous vortexing, the samples were centrifuged for $30 \mathrm{~min}$ at $4^{\circ} \mathrm{C}$ and $3000 \mathrm{~g}$. The supernatants were filtered through a $0.45 \mu \mathrm{m}$ filter device.

\section{HPLC}

Fifty microliters of the perchloric acid extracts was injected into the HPLC system, consisting of the BioRad 2700 solvent delivery system, AS-100 HRLC automatic sampling system, 1801 UV Monitor (BioRad, Hercules, CA, USA), and a $300 \times 8 \mathrm{~mm}$ organic acid resin column (Goehler HPLC-Analysentechnik, Chemnitz, Germany). The elution was performed with $5 \mathrm{mM} \mathrm{H}_{2} \mathrm{SO}_{4}$ with a flow rate of $0.8 \mathrm{ml} / \mathrm{min}$. Measurements were performed at ambient temperature, detecting changes in absorbance at a wavelength of $210 \mathrm{~nm}$. All chemicals were purchased from Sigma or Roth (Carl Roth $\mathrm{GmbH}+$ Co. KG, Karlsruhe, Germany). Perchloric acid extracts that were prepared for HPLC were also used for the photometric assays.

\section{PDH Assay}

Chemicals and enzymes for photometric assays were purchased from Sigma, Roche, or Roth. The $\mathrm{PDH}$-based photometric assay solution consisted of $0.25 \mathrm{M}$ MOPS-Cl (pH 7.4 at $30^{\circ} \mathrm{C}$ ), $5 \mathrm{mM} \mathrm{MgCl}_{2}$, $1 \mathrm{mM} \mathrm{CaCl}_{2}, 2 \mathrm{mM}$ TPP, $0.25 \mathrm{mM} \mathrm{CoA}, 6 \mathrm{mM} \mathrm{NAD}^{+}$, and $3 \mathrm{mM}$ L-cysteine. For determination of pyruvate, $10 \mu \mathrm{l}$ of perchloric acid extracts was added to $75 \mu \mathrm{l}$ of the assay solution, and absorbance at $340 \mathrm{~nm}$ was measured with a microplate reader (BioRad, Model $3550-U V)$. Twenty microliters of PDH $(0.6 \mathrm{U} / \mathrm{ml})$ was added, and absorbance at $340 \mathrm{~nm}$ was determined in 5 min intervals. When no further increase of absorbance occurred, the respective $\Delta \mathrm{A} 340$ was registered for standards and samples. 


\section{LDH Assay}

This assay utilizes the decrease in absorbance at $340 \mathrm{~nm}$, as NADH is metabolized. The LDH-based photometric assay solution consisted of $0.1 \mathrm{M}$ TEA (triethanolamine, $\mathrm{pH} 7.6$ ), $4 \mathrm{mM} \mathrm{MgCl}_{2}, 30 \mathrm{mM} \mathrm{KCl,}$ and $0.3 \mathrm{mM}$ NADH. Determination of pyruvate followed as described in the PDH-based assay. When no further decrease of absorbance was detectable, the respective $\Delta \mathrm{A} 340$ was acquired for standards and samples.

\section{Results}

\section{Stability of Pyruvate}

The chemical stability of pyruvate was investigated, as tissue sections have to be heated to inactivate endogenous enzymes before the induction of the bioluminescence reaction. Furthermore, the preparation of extracts of tissues and biopsies includes sonification of the specimens. Pyruvate was therefore determined in aqueous solutions by the photometric LDH assay following heat or sonification compared to untreated controls. Figure 2a shows that heat autoclaving of a sodium pyruvate solution for $30 \mathrm{~min}$ had no degrading effect on this metabolite. Correspondingly, sonifying standard solutions for $30 \mathrm{~min}$ had no decomposing effect on pyruvate (Figure 2b).

\section{Pyruvate-Dependent Bioluminescence}

To ensure equal processing of tissue samples and standards, calibration standards were prepared from liver homogenates. These can be heat-inactivated in a way identical to that used for sections of biopsies or other sample tissue. Figure 3 shows a representative calibration curve of the pyruvate-dependent bioluminescence using liver homogenate standards. The reaction is linear between 0 and $5.0 \mu \mathrm{mol} / \mathrm{g}$. Linear regression through the data points makes it possible to convert light intensity from unknown section samples into absolute concentrations in $\mu \mathrm{mol}$ pyruvate per g tissue (dry weight). Registration of light emission occurs within a defined time interval with regard to the reaction kinetic (Figure 4). Measuring pyruvate via bioluminescence is not an end point test, as longer integration times would be associated with increased lateral substrate diffusion and thus with a decrease in spatial resolution. ${ }^{15,18}$ Acquisition and integration of light intensity for $1 \mathrm{~min}$ and $45 \mathrm{~s}$ after a $10 \mathrm{~s}$ preincubation time yielded the data shown in Figure 3.

Bioluminescence intensity was proportional to the total amount of pyruvate entering the biochemical detection procedure, that is, to the metabolite content of the tissue section. This was demonstrated by changing the section thickness of standards between 5 and $25 \mu \mathrm{m}$ at a constant pyruvate
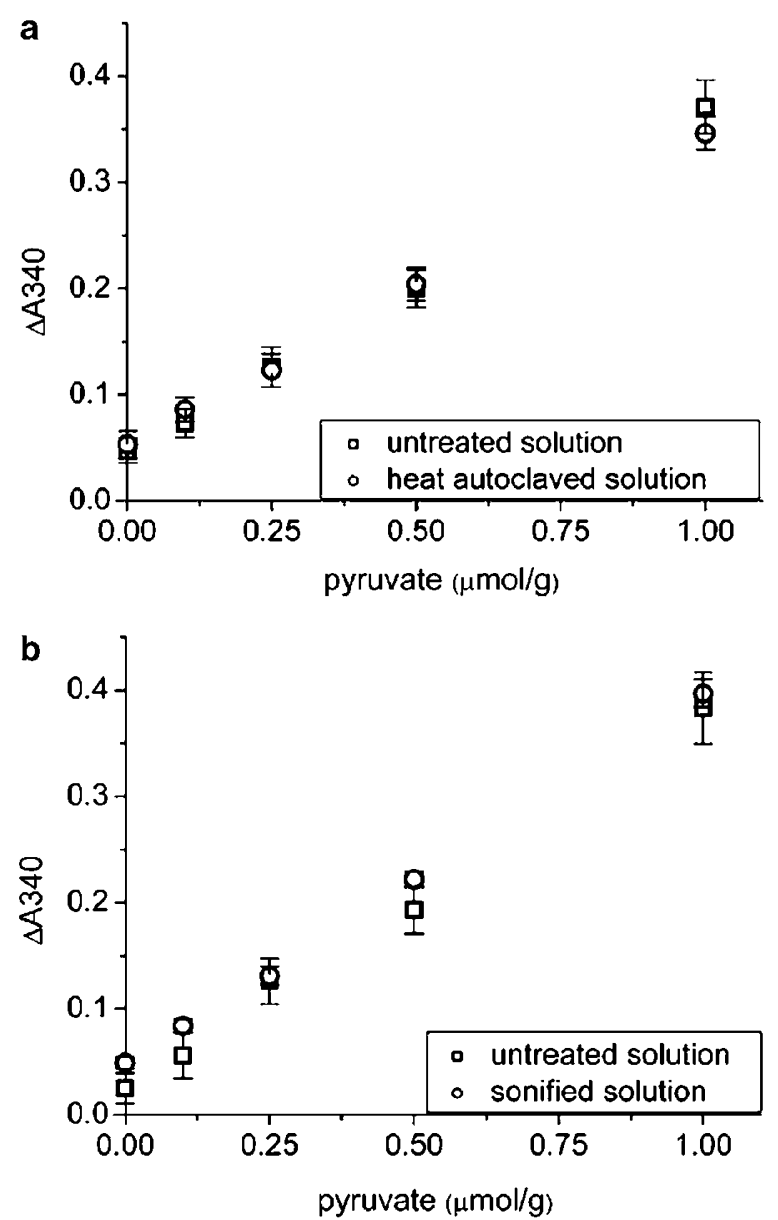

Figure 2 Stability of pyruvate. Relative absorbance $(\Delta \mathrm{A} 340)$ as a function of pyruvate concentration assayed photometrically via the LDH assay. (a) Heat stability and (b) mechanical stability. Mean values from (a) sextuples \pm s.d. and (b) triplets \pm s.d. are shown for untreated (square) and treated (circle) solutions.

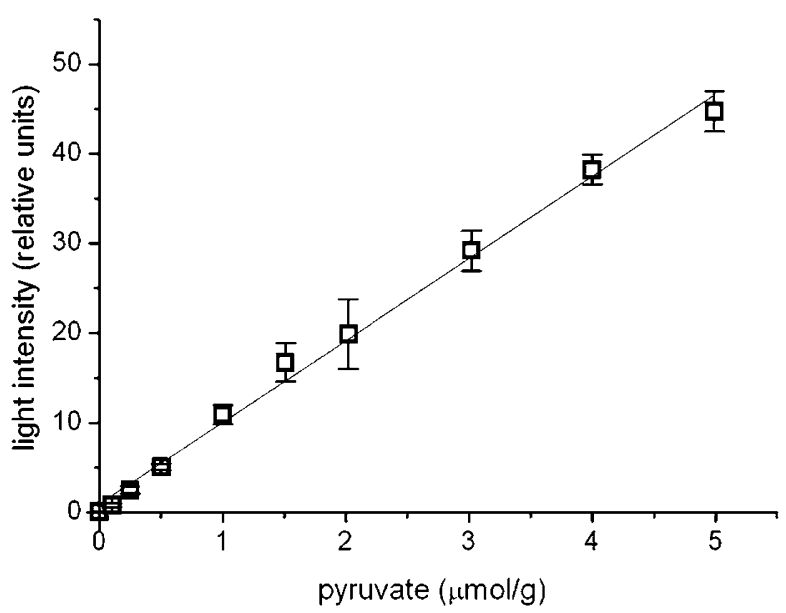

Figure 3 Pyruvate-dependent bioluminescence using liver homogenate standards. Representative calibration curve of pyruvatedependent bioluminescence from liver homogenate standards: light intensity \pm s.d. using $20 \mu \mathrm{m}$ sections of frozen liver homogenate standards (relative units) as a function of pyruvate concentrations in $\mu \mathrm{mol} / \mathrm{g}$. Linear regression: $y=0.858+9.16 x$; $r=0.997 ; P<0.0001 ; n=3$ for all concentrations. 


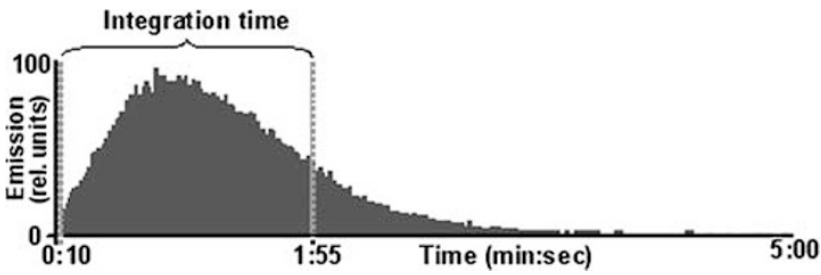

Figure 4 Reaction kinetics of pyruvate-dependent bioluminescence. Relative emission intensity of the pyruvate-dependent bioluminescence mixture within $5 \mathrm{~min}$. A $1.5 \mu \mathrm{mol} / \mathrm{g}$ standard section $(20 \mu \mathrm{m})$ was used for measurement using the pyruvate enzyme mixture. The integration time is indicated in the figure.

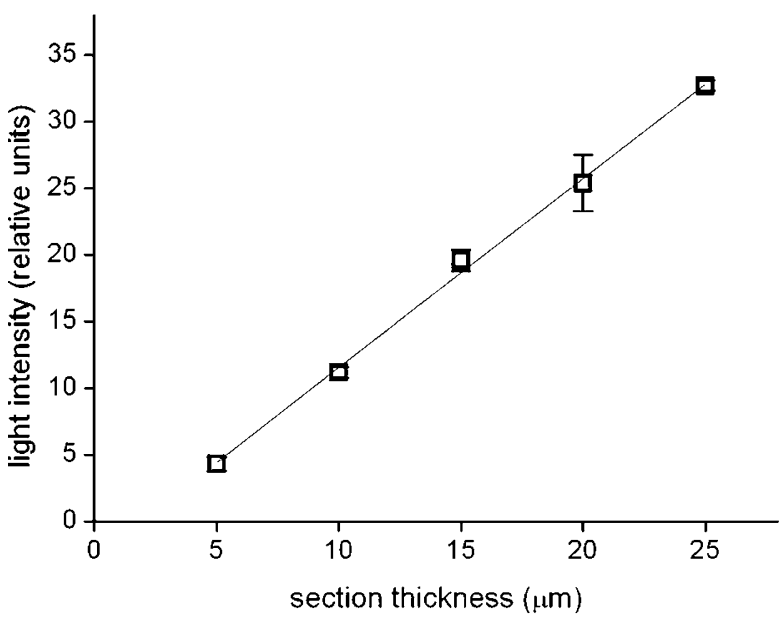

Figure 5 Bioluminescence intensity as a function of section thickness at a constant pyruvate concentration. All measurements were performed using sections from one standard and the same enzyme solution at the same day. Mean values from triplets \pm s.d are shown. Linear regression: $y=-2.66+1.42 x ; r=0.999$; and $P<0.0001$

concentration. Figure 5 shows that a linear correlation exists between section thickness and bioluminescence intensity.

We conclude from the data shown in Figures 3 and 5 that, at a constant section thickness, light intensity produced by the novel bioluminescence assay is proportional to the initial pyruvate concentration. The assay allows for the determination of pyruvate concentrations in sections of frozen tissue in the range of $0-5.0 \mu \mathrm{mol} / \mathrm{g}$ tissue (dry weight). Variation coefficients from multiple measurements were less than $5 \%$ in all cases. The minimal detectable amount is 0.02 pmol based on a $20 \mu \mathrm{m}$ thick cryosection with an area of $1 \mathrm{~cm}^{2}$.

\section{Quantitation of Pyruvate in Tumor Biopsies of Human Head and Neck Squamous Cell Carcinomas}

For the determination of lactate and pyruvate in an HNSCC xenograft (courtesy of Michael Baumann, Technical University of Dresden, Germany), serial cryosections were prepared of the rapidly frozen tissue (Figure 6). HE stainings were used to discriminate between carcinoma (TU) and surrounding normal tissue, such as skeletal muscle (MU) and skin (SK). Before the bioluminescence reaction, a brightfield image was taken of the sections for later colocalization of the bioluminescence images. Before sectioning, two pinpricks were applied to the biopsy in order to provide landmarks for a correct overlay of corresponding images (see holes in the sections shown in Figure 6). Figure 6 illustrates the feasibility and potency of quantitative and structure-associated mapping of lactate, pyruvate, and the $\mathrm{L} / \mathrm{P}$ ratio using quantitative bioluminescence with a spatial resolution on a microscopic level.

Based on the correct overlay of the lactate and pyruvate images, a map of L/P ratios was derived from the division of each pixel value of lactate by the corresponding pixel value of pyruvate. For statistics, these three images were evaluated by producing frequency distribution histograms of the respective pixel data for the whole section and for the selected histological areas, as depicted in Figure 7. Statistical parameter values, such as means, medians (Med), and standard deviations (s.d.), were obtained from these distributions.

As demonstrated in Figures 6 and 7, lactate and pyruvate concentrations were higher and scattered over a wider range in tumor vs normal tissue. Pixel values for $\mathrm{L} / \mathrm{P}$ ratios were higher in tumor tissue compared to skin and muscle, but were more uniform in malignant compared to normal tissue. These findings provide evidence for the novel technique to enable studies on larger cohorts of solid tumors with regard to carbohydrate metabolism, redox state, and therapeutic resistance.

\section{Verification and Recovery}

\section{Non-bioluminescent pyruvate assays}

Non-bioluminescent assays were used to verify pyruvate measurements with bioluminescence. For this purpose, perchloric acid extracts of liver homogenate standards and reference samples were prepared as described in Materials and methods. The extracts were analyzed by HPLC and two different enzymatic photometric assays.

\section{HPLC}

Pyruvate concentrations of liver homogenate standards and reference samples were determined via anion exchange chromatography. Peaks were identified by the retention time following the addition of known amounts of pyruvate to the samples. In three independent experiments, there was a highly significant linear correlation $(r=0.999 ; P<0.0001)$ between given pyruvate concentrations in the standards and the respective peak areas (data not shown).

\section{Photometric assays}

$\mathrm{LDH}$ and $\mathrm{PDH}$ were used in two independent photometric assays to verify the novel biolumin- 
a

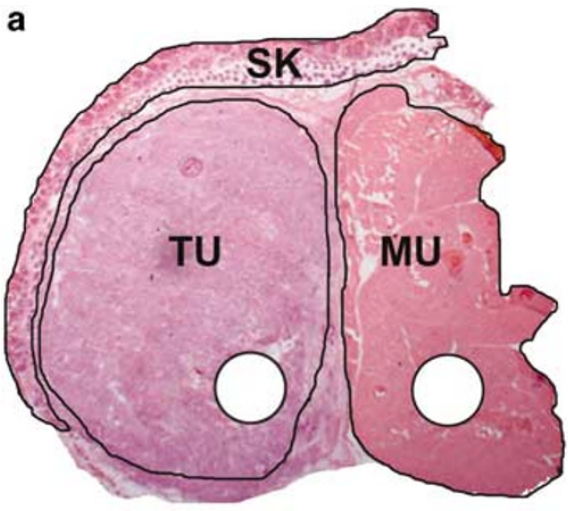

b

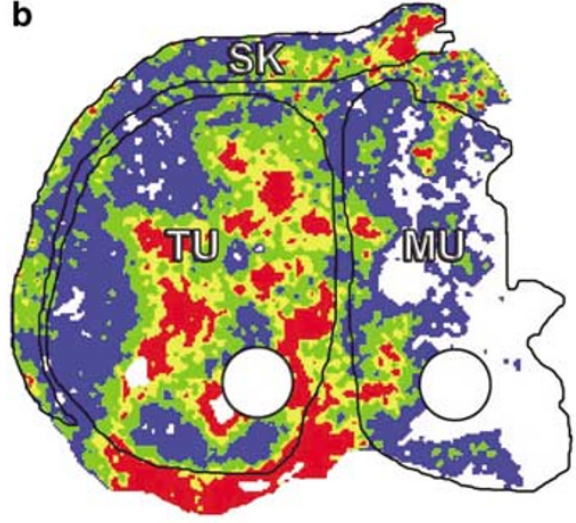

$1 \mathrm{~mm}$

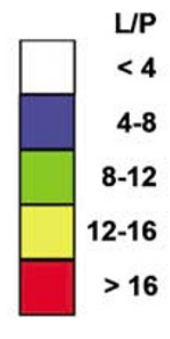

C

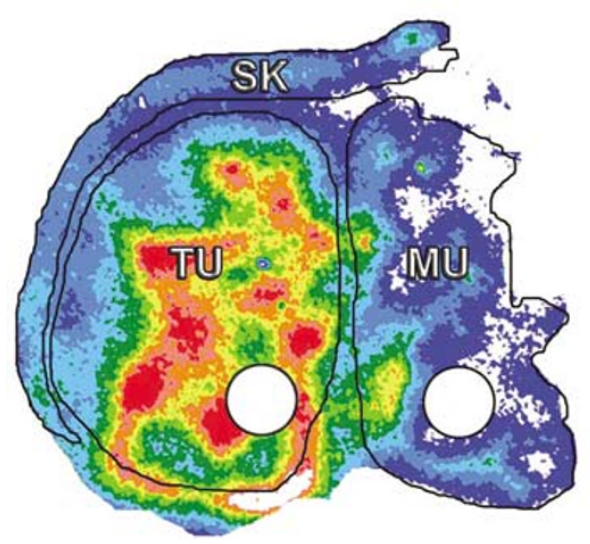

d

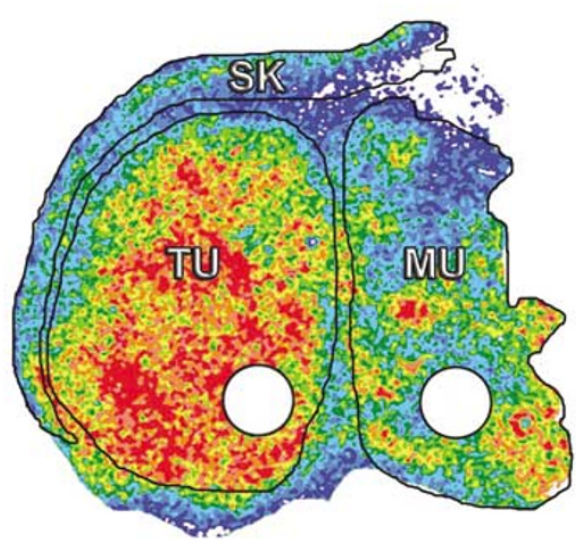

Figure 6 Imaging bioluminescence of pyruvate, lactate, and L/P ratio in a human head and neck squamous cell carcinoma (HNSCC) xenografted in a nude mouse (courtesy of Michael Baumann, Dresden, Germany). Sequential cryosections: (a) hematoxylin and eosin (H\&E) staining $(10 \mu \mathrm{m})$ as well as color-coded distributions of (b) lactate-to-pyruvate $(\mathrm{L} / \mathrm{P})$ ratios and of concentrations of (c) lactate $(16 \mu \mathrm{m})$ and $(\mathbf{d})$ pyruvate $(20 \mu \mathrm{m})$. Pinpricks within the biopsy provide landmarks (ie two holes) for a correct overlay of corresponding images. For structure-associated evaluation, different histological areas were delineated, that is, tumor tissue (TU), skeletal muscle (MU), and skin (SK).

escence assay. The PDH enzyme assay is the basic enzyme reaction of the bioluminescence reaction mixture. Extracts of standards and reference samples were analyzed in triplicate in end point tests at $340 \mathrm{~nm}$. In analogy to the HPLC measurements, calibration curves of liver homogenate standard extracts, resulting from the $\mathrm{PDH}$ or LDH photometric assay, were linear with statistical parameters of $r=0.999$ and $P<0.0001$ (data not shown).

Recovery of the pyruvate-dependent bioluminescence assay was determined in 10 reference samples of liver homogenates. These were tested with the bioluminescence technique as well as by the reference methods HPLC and enzymatic assays (Table 1). Reference samples were prepared in the same way as liver homogenate standards and were measured in triplicate. The novel bioluminescence assay for pyruvate exhibits a recovery with a variance of $\leq 15 \%$ from given values in all reference samples analyzed. Major deviations occurred for sample 1 in both the PDH and LDH assay (Table 1). As HPLC measurements using the same extract of sample 1 did not show such a large deviation, this most likely indicates a limited sensitivity of the enzymatic assays in the lower concentration range.

\section{Discussion}

Metabolite imaging with bioluminescence has been applied with success in a variety of biological systems, such as soy bean seedlings, ${ }^{19}$ complex 3D cell cultures, ${ }^{20}$ numerous normal organs, and surgical wounds from laboratory animals, ${ }^{21}$ as well as in various experimental tumors and human solid tumors in the clinic (reviewed by Walenta et $a l^{16}$ ). Till now, bioluminescence imaging has been possible for the detection of ATP, glucose, lactate, 
lactate
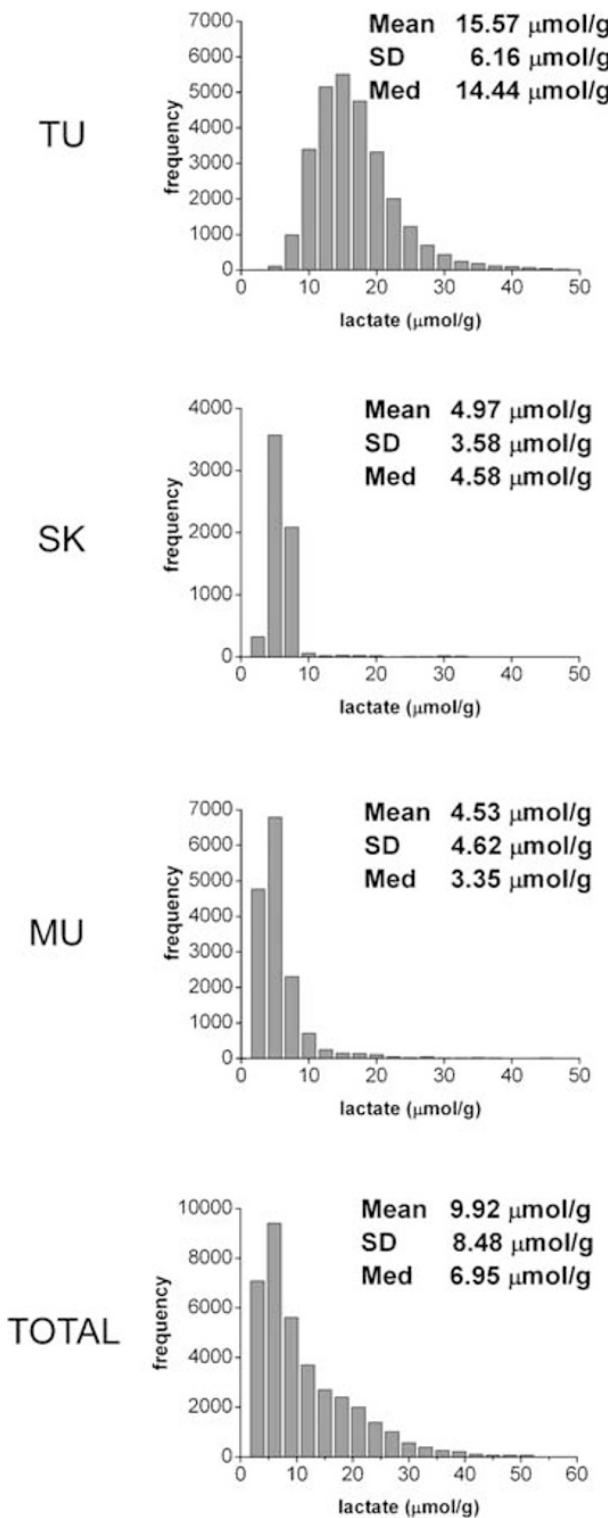

pyruvate
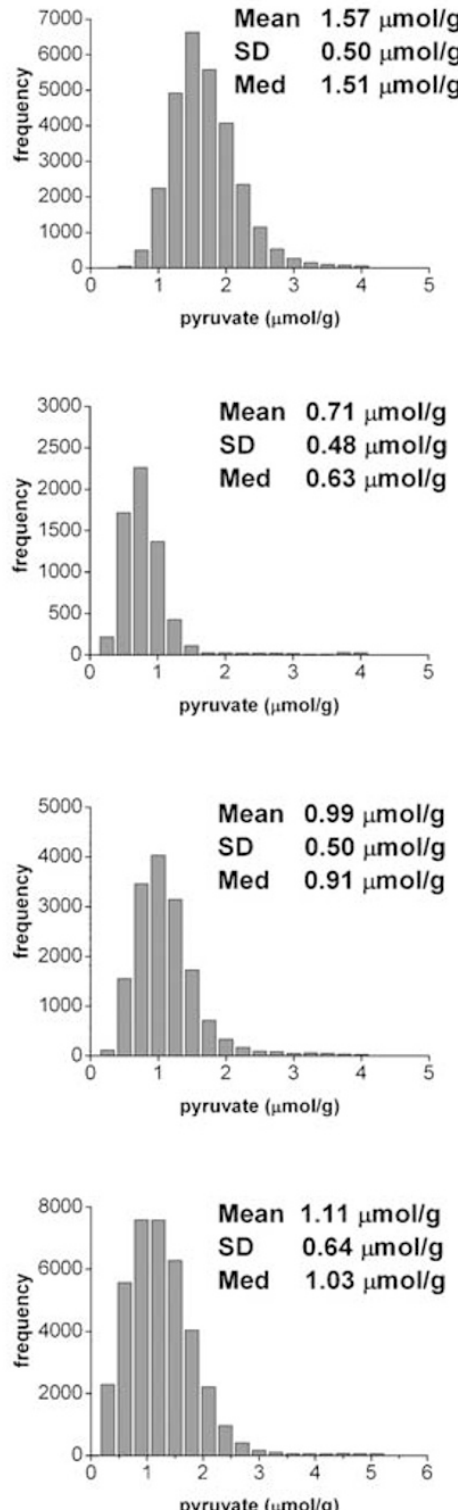
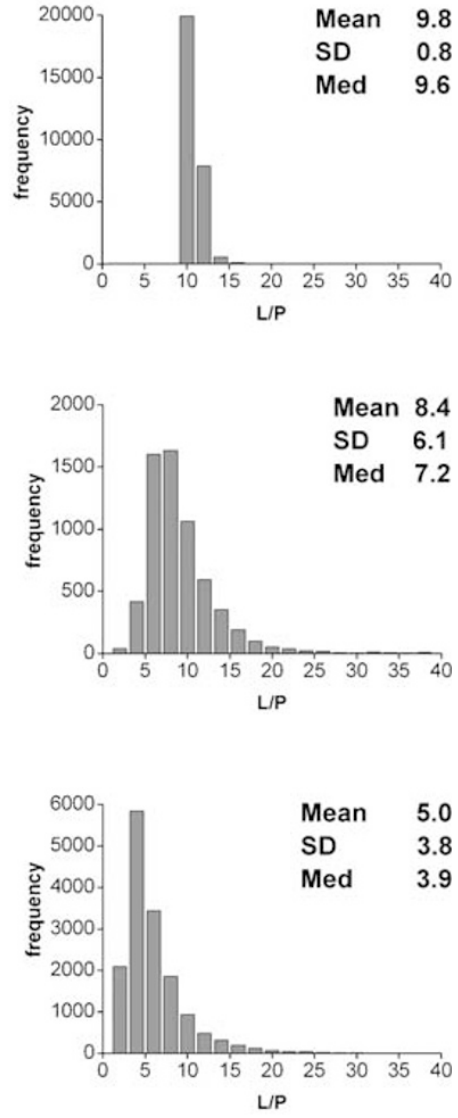

Mean 8.4

SD 6.1

2

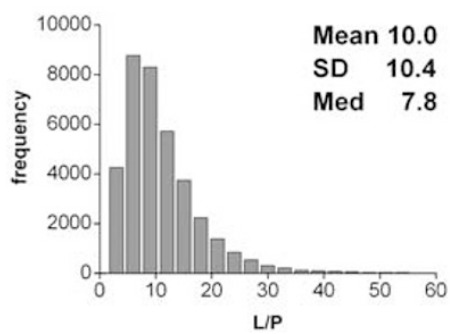

Figure 7 Frequency distribution histograms and statistics of pixel values for lactate, pyruvate, and L/P ratios measured with bioluminescence in the tumor presented in Figure 6. Histograms are depicted for lactate (first column), pyruvate (center column), and L/P ratios (third column) in tumor tissue (TU), skin (SK), muscle (MU), and the whole tissue section (TOTAL). Statistical evaluation resulted in values for the mean, standard deviation (s.d.), and the median (Med).

sucrose, and glycogen. ${ }^{11,18,19}$ Although accumulation of lactate may be indicative of hypoxia under certain conditions, the $\mathrm{L} / \mathrm{P}$ ratio is a much more direct indicator of the redox state of cells or tissue. Assessment of this information may be particularly important in solid malignant tumors where the redox state is related to the growth behavior and therapeutic resistance. ${ }^{22}$ Thus, the methodological development introduced by this report not only supplements the pre-existing bioluminescence imaging technique but also fills a gap in the accessibility of metabolism in healthy and aberrant tissue. We have shown that bioluminescence imaging can be used now for the determination of the $\mathrm{L} / \mathrm{P}$ ratio in snap-frozen biological tissue.

The pyruvate molecule was shown to remain intact during heating and sonifying, which is important, as the samples for bioluminescence need to be heat-inactivated and those used for non-bioluminescent assays have to be sonified. We succeeded in creating a pyruvate-dependent bioluminescence assay that enables the determination of absolute pyruvate concentration values in frozen tissue sections in the range of $0-5.0 \mu \mathrm{mol} / \mathrm{g}$ tissue 
Table 1 Pyruvate concentrations ( $\mu \mathrm{mol} / \mathrm{g})$ measured by different assays

\begin{tabular}{|c|c|c|c|c|c|c|c|c|c|}
\hline \multirow{2}{*}{$\begin{array}{l}\text { Sample } \\
\text { number }\end{array}$} & \multirow{2}{*}{$\begin{array}{c}\text { Reference } \\
\text { sample } \\
\mu m o l / g \\
\text { (given value) }\end{array}$} & \multicolumn{2}{|c|}{ Bioluminescence } & \multicolumn{2}{|c|}{ HPLC } & \multicolumn{2}{|c|}{ PDH assay } & \multicolumn{2}{|c|}{ LDH assay } \\
\hline & & $\mu \mathrm{mol} / \mathrm{g}$ & $\begin{array}{l}\% \text { deviation } \\
\text { of recovery }\end{array}$ & $\mu \mathrm{mol} / \mathrm{g}$ & $\begin{array}{l}\text { \% deviation } \\
\text { of recovery }\end{array}$ & $\mu \mathrm{mol} / \mathrm{g}$ & $\begin{array}{l}\text { \% deviation } \\
\text { of recovery }\end{array}$ & $\mu \mathrm{mol} / \mathrm{g}$ & $\begin{array}{c}\% \text { deviation of } \\
\text { recovery }\end{array}$ \\
\hline 1 & 0.18 & 0.17 & -6 & 0.16 & -11 & 0.30 & ${ }^{*} 68$ & 0.25 & *41 \\
\hline 2 & 0.92 & 0.78 & -15 & 0.83 & -10 & 0.94 & 2 & 0.87 & -5 \\
\hline 3 & 1.76 & 1.78 & 1 & 1.76 & 0 & 1.77 & 1 & 1.73 & -2 \\
\hline 4 & 1.02 & 1.03 & 1 & 0.98 & -4 & 1.03 & 1 & 0.94 & -8 \\
\hline 5 & 1.61 & 1.52 & -6 & 1.60 & -1 & 1.59 & -1 & 1.59 & -1 \\
\hline 6 & 0.23 & 0.26 & 13 & 0.21 & -7 & 0.19 & $*-18$ & 0.22 & -3 \\
\hline 7 & 1.47 & 1.69 & 15 & 1.51 & 3 & 1.28 & -13 & 1.45 & -1 \\
\hline 8 & 0.51 & 0.48 & -6 & 0.50 & -2 & 0.44 & -14 & 0.47 & -9 \\
\hline 9 & 1.83 & 2.00 & 9 & 1.89 & 3 & 1.68 & -8 & 1.82 & 0 \\
\hline 10 & 0.99 & 1.02 & 3 & 0.99 & 0 & 0.96 & -3 & 1.00 & 2 \\
\hline
\end{tabular}

The novel pyruvate bioluminescence method, the reference methods HPLC, and the two photometric assays were used to analyze pyruvate concentrations in reference samples.

With the exception of three experiments $(*)$, the relative deviation of each data point compared to reference samples was $\leq 15 \%$.

(dry weight). Light emission produced by the novel approach is proportional to the pyruvate concentration. The intensity of light was dependent not only on the concentration within the section but also on the section thickness in a proportional manner. Thus, it is important to prepare cryosections of identical thickness throughout measurement and calibration. The enzyme mixture tested allows for both sensitive detection even of very low amounts of pyruvate and assessment of its spatial distribution on a microscopical level in relation to the tissue structure. In contrast to other methods, such as HPLC or photometric assays, only very small sample volumes and no chemical extraction procedures are required for metabolite determination. Furthermore, the assay exhibits a higher sensitivity than the photometric tests investigated. In the current study, the application of the new assay has been illustrated in a tissue sample of HNSCC xenografts containing tumor tissue, muscle, and skin.

The data demonstrate the feasibility and potency of the novel bioluminescence assay for the detection of pyruvate in cryosections. Further investigations will be performed to compare pyruvate concentrations in normal and tumor tissues in a systematic way. Combination of imaging bioluminescence of ATP, glucose, lactate, ${ }^{11}$ and pyruvate will enable us to assess and characterize the metabolic milieu of individual tumors more comprehensively. As the $\mathrm{L} / \mathrm{P}$ ratio reflects the redox state of the cell, it will be important to analyze the relationship between these two metabolites in tumors on a large scale. Pyruvate as well as lactate is known to have radical scavenger function. ${ }^{13,14}$ Thus, information on these two metabolites within tumors could permit predictions concerning radioresistance/radiosensitivity of tumors ${ }^{23}$ and may open up ways of more efficient therapeutic attacks on malignancies.

\section{Acknowledgements}

This work was supported by the Stiftung RheinlandPfalz für Innovation, grant \# 15202-386261/606. We thank Tobias Leidig for scientific advice on data analysis.

\section{References}

1 Benoist JF, Alberti C, Leclercq S, et al. Cerebrospinal fluid lactate and pyruvate concentrations and their ratio in children: age-related reference intervals. Clin Chem 2003;49:487-494.

2 Fukui H, Taniguchi S, Ueta Y, et al. Enhanced activity of the purine nucleotide cycle of the exercising muscle in patients with hyperthyroidism. J Clin Endocrinol Metab 2001;86:2205-2210.

3 Constantin-Teodosiu D, Peirce NS, Fox J, et al. Muscle pyruvate availability can limit the flux, but not activation, of the pyruvate dehydrogenase complex during submaximal exercise in humans. J Physiol (London) 2004;561:647-655.

4 Ong HY, O’Dochartaigh CS, Lovell S, et al. Gas exchange responses to constant work-rate exercise in patients with glycogenosis type V and VII. Am J Respir Crit Care Med 2004;169:1238-1244.

5 Marsac C, Benelli C, Desguerre I, et al. Biochemical and genetic studies of four patients with pyruvate dehydrogenase E1 alpha deficiency. Hum Genet 1997;99:785-792.

6 Sahlin K, Katz A, Henriksson J. Redox state and lactate accumulation in human skeletal muscle during dynamic exercise. Biochem J 1987;245:551-556.

7 DiMauro S, Lamperti C. Muscle glycogenoses. Muscle Nerve 2001;24:984-999.

8 Minniti G, Cerone R, De Toni E. Determination of lactic acid, pyruvic acid, and ketone bodies in serum and cerebrospinal fluid by HPLC. Am Clin Lab 2001;20:21-23.

9 Rosenberg JC, Rush BF. An enzymatic-spectrophotometric determination of pyruvic and lactic acid in blood. Methodologic aspects. Clin Chem 1966;12: 299-307. 
10 O'Donnell-Tormey J, Nathan CF, Lanks K, et al. Secretion of pyruvate. An antioxidant defense of mammalian cells. J Exp Med 1987;165:500514.

11 Mueller-Klieser W, Walenta S. Geographical mapping of metabolites in biological tissue with quantitative bioluminescence and single photon imaging. Histochem J 1993;25:407-420.

12 Roslin M, Henriksson R, Bergstrom P, et al. Baseline levels of glucose metabolites, glutamate and glycerol in malignant glioma assessed by stereotactic microdialysis. J Neurooncol 2003;61:151-160.

13 Groussard C, Morel I, Chevanne M, et al. Free radical scavenging and antioxidant effects of lactate ion: an in vitro study. J Appl Physiol 2000;89: 169-175.

14 Brand KA, Hermfisse U. Aerobic glycolysis by proliferating cells: a protective strategy against reactive oxygen species. FASEB J 1997;11:388-395.

15 Walenta S, Wetterling M, Lehrke M, et al. High lactate levels predict likelihood of metastases, tumor recurrence, and restricted patient survival in human cervical cancers. Cancer Res 2000;60:916-921.

16 Walenta S, Schroeder T, Mueller-Klieser W. Lactate in solid malignant tumors: potential basis of a metabolic classification in clinical oncology. Curr Med Chem 2004;11:2195-2204.
17 Brizel DM, Schroeder T, Scher RL, et al. Elevated tumor lactate concentrations predict for an increased risk of metastases in head-and-neck cancer. Int J Radiat Oncol Biol Phys 2001;51:349-353.

18 Levin M, Leppanen O, Evaldsson M, et al. Mapping of ATP, glucose, glycogen, and lactate concentrations within the arterial wall. Arterioscler Thromb Vasc Biol 2003;23:1801-1807.

19 Borisjuk L, Walenta S, Rolletschek H, et al. Spatial analysis of plant metabolism: sucrose imaging within Vicia faba cotyledons reveals specific developmental patterns. Plant J 2002;29:521-530.

20 Walenta S, Doetsch J, Mueller-Klieser W, et al. Metabolic imaging in multicellular spheroids of oncogene-transfected fibroblasts. J Histochem Cytochem 2000;48:509-522.

21 Walenta S, Schroeder T, Mueller-Klieser W. Metabolic mapping with bioluminescence: basic and clinical relevance. Biomol Eng 2002;18:249-262.

22 Kinnula VL, Crapo JD. Superoxide dismutases in malignant cells and human tumors. Free Radic Biol Med 2004;36:718-744.

23 Quennet V, Yaromina A, Zips D, et al. Tumor lactate content predicts for response to fractionated irradiation of human squamous cell carcinomas in nude mice. Radiother Oncol 2006; 12 September [Epub ahead of print] PMID: 16973228. 